PPPL- -2634

DE89 014606

\section{DISCLAIMER}

This report was prepared as an account of work sponsored by an agency of the Uniled Stute Government. Neither the United States Guvernment nor any agency thereof, nor any of their employes, makes any wareanty, express or implied, or assumes any legal liability or responsibility for the accuracy, completesess, or uselulness of any information, apparatus, product, or process disclosed, or represents that its use would not infringe privately owned rights. Reference herein to any specific commercial product, process, or service by trade name, trademart, manufacturer, or otherwise does not necessarily constitute of imply its endorsement, recommendation. or favoring by the United States Government or any agency thereol. The views and opinions of authors expressed herein do not necessarily state or reflect those of the United States Govemment or any agency thereof.

\title{
Tokamak electrical conductivity modific $\perp$ by electrostatic trapping in the applied electric fleld
}

\author{
C.S. Chang* \\ Princeton Plasma Physics Laboratory \\ Princeton University \\ Princeton, NJ 08543
}

Passing electrons traveling in the same direction as the applied Ohmic electric field, near the trapped-passing boundary, can get electrostatically trapped by the applied Ohmic potential, reverse their parallel flow direction, and become passing particles traveling in the opposite direction. The number of these electrons is proportional to the applied voltage and is small. But, the amount of change in their time-averaged parallel velocity, by reversing their flow direction, is about twice the average parallel velocity of the barely passing electrons and is large. Hence, these electrostatically trapped particles can significently contribute to the Ohmic electrical current. The new electrical conductivity is calculated in the banana regime using a pitch angle scattering operator, and the values are found to be significantly enhanced from previous neoclassical theories.

\footnotetext{
-Courant Inatitute of Mathematical Sciences, New York University, and Korea Inatitute of Technology
} 
Establishing an accurate theoretical expression for electrical conductivity is an important task in understanding tokamak plasma behavior. Without an accurate electrical conductivity calculation, one cannot have confident analyses of current profile and, thus, of basic plasma equilibrium and stability. Despite the turbulent behavior of tokamak plasmas, it has been evidenced experimentally that the electrical conductivity in tokamaks may not be affected by plasma turbulence, and thus neoclassical analysis is a reliable tool for the subject.

In previous analyses, ${ }^{1-3}$ it was shown that electrical conductivity values in a tokamak were significantly reduced from the result of Spitzer" due to the electron trapping effect in the inhomogeneous magaetic field and also due to the collisional drag effect by the trapped electrons. However, in order to simplify the analyses, the previous theories used an approximation based on the conservation of particle kinetic energy, instead of conservation of the total energy, which includes the applied potential energy. Since the applied Ohmic voltage is usually small compared to the particle's kinetic energy, the approximation is not expected to yield much error for the particles deeply passing or trapped. But, for the particles near the trapped-passing boundary, their parallel kinetic energy becomes small near the maximum- $B$ point and the modification of the particle orbit by the applied voltage becomes significant. As a result, some of the passing electrons traveling in the same direction as the applied Ohmic electric field $\left(\vec{E}_{O A_{m}}\right)$ near the trapped-passing boundary are forced by the applied electric field to turn around and become part of the oppositely traveling 
passing electrons (see Fig. 1). We call these particles "Chmically reversing" electrons in the present work. The motion of these electrons is characterized by an applied-electric-field driven electron flux in velocity space across the trapped-passing boundary, which crosses the boundaries from the costreaming (relative to $\vec{E}_{O h m}$ ) passing region to the trapped region and then to the counter-streaming passing region. This extra particle flux (eventually balanced by the collisional counter flux) across the trapped-passing boundary is found to generate an additional Obmic electrical current and enhance the neoclassical conductivity. Previous analysas did not allow for such a particle motion due to the approximation that the particle kinetic energy is a constant of motion.

In Spitzer's analyses of electrical conductivity in a homogeneous magnetic field geometry, this Ohmically reversing particle effect was included naturally. However, the average parallel (to $\vec{E}_{O h m}$ ) velocity of the Ohrmically reversing electrons was vanishingly small in a homogeneous magaetic field, and thus they did not contribute to the electrical conductivity. The contribution from the Ohmically reversing electrons becomes sįnificant only in an inhomogeneous magnetic field geometry, where the time-averaged parallel velocities of those particles do not vanish, but are rather large. It is, therefore, important to include these particles in the analyses to obtain correct values for electrical conductivity in an inhomogeneous magnetic field. In previous neoclassical theories, which used the Spitzer equation as the basis of analyses, the effect of these Ohnically reversing electrons was implicitly included in the Spitzer function, but only to the extent of 
homogeneous magnetic field problems where such an effect gave negligible contribution.

Qualitative estimation of the amount of additional current generation by these Ohmically reversing electrons can tse easily obtained. The fraction of the Ohmically reversing electrons is roughly the thickeness $\Delta v_{\|} / v$ of the pitch angle layer, near the trapped-passing boundary, occupied by these particles. If the electrons are within this layer, the amount of electrostatic deceleration in a bounce time is large enough to force them to reverse their flow direction gear the maximum Mod-B point, satiffying $m \Delta v_{\|} \sim e E_{\|} \eta$. Thus the number density $\Delta n$ is roughly given as $\Delta n \sim\left(e E_{\|} \tau_{b} / m v\right)$. Since the magnitude of the parallel velocity change for these particles dear the trapped-passing boundary is $(r / R)^{1 / 2} v$, the rate of current generation is $d / d t \sim(e \Delta n / \pi)(r / R)^{1 / 2} v \sim\left(n e^{2} / m\right)(r / R)^{3 / 2} E_{\|}$. This current generation will eventually have to be balanced by Coulomb collisions, and we get the relation $(r / R)^{1 / 2}\left(n e^{2} / m\right) E_{\|} \sim \nu_{c} j$, where $\nu_{\varepsilon}$ is the Coulomb $90^{\circ}$-collision frequency. Hence the amount of current generated by the Ohmically reversing electrons is $j \sim(r / R)^{1 / 2}\left(n e^{2} / m \nu_{\mathrm{c}}\right) E_{\mathrm{l}}$, which is of the same order of magnitude as the usual neoclassical reduction current.

We define $\varepsilon$ and $\lambda$ as the total energy and the magnetic moment normalized to the kinetic energy $W$ per unit mass, respectively: $\varepsilon=W+$ $q \phi / m, \lambda=(\mu / W)=v_{\perp}^{2} / 2 W B$. Then $\varepsilon$ is a constant of motion, but $\lambda$ is not. If we define $\theta$ as a poloidal angle and $\sigma \equiv \eta_{\|} /\left|\nu_{\|}\right|$, the electron motion in $(\lambda, \sigma, \theta)$-space is as described in Fig. 2. We can from Fig. 2 that the passing electrons crossing the usual, purely magnetic, trapped-passing 
boundary (dotted line) from the $\sigma=1$ side bounce back and cross the other trapped-passing boundary at the $\sigma=-1$ side into the counter-streaming passing regime. The angle at which the Ohmically reversing electrons meet the usual trapped-passing boundary is a function of particle kinetic energy and thus the combined electro-magnetic trapped-passing boundary cannot be defined uniquely. In other words, the higher energy electron motions are less affected and the lower energy ones are more affected. For this reason, we will still keep the usual purely magnetic definition of trapped-passing boundary in the present work.

Since the Ohmically reversing motions can be characterized by the rapid change of particle pitch angles into and out of the trapped region (in the usual sense), in order to study their effect on the electrical current, we need to calculate accurately the fast exchange rate of the particles between the trapped and passing regions. For this purpose, it is more convenient to treat the trapped and passing particles as separate species, but collisionally shaped together into a Maxwellian, to the lowest order. Notice here that as far as other dissipational processes (other than the Coulomb collisions) are concerned, trapped and passing particles respond differently and they are two different groups of particles.

We note here that the present problem may also be hardled without separating the trapped and passing electrons into two different species, as long as the fast particle flow across the trapped-passing boundary is evaluated accurately outside of the drift kinetic equation and used, as a boundary condition, in the determination of the perturbed distribution 
function. But, as will be discussed later, such an approach may be more complicated than the one used in the present analyses.

The usual drift-kinetic equation is written as

$$
\vec{v}_{\|} \cdot \nabla f_{\alpha}=C\left(f_{\alpha}\right)+\nabla \cdot \vec{\Gamma}_{\alpha},
$$

where $\alpha=t$ for trapped electrons and $\alpha=p$ for passing electrons, and $\Gamma_{a}$ is the particle flux in the velocity space driven by the applied electric field. In terms of $v$ and $\xi=\eta_{\eta} / v, \nabla \cdot \vec{\Gamma}$ can be expressed as

$$
\nabla \cdot \vec{\Gamma}=\frac{e E_{\| 1}}{m}\left(\frac{v_{l}}{v} \frac{\partial f_{a}}{\partial v}+\frac{1-\xi^{2}}{v} \frac{\partial f_{a}}{\partial \xi}\right),
$$

where $E_{\|}$is the parallel component of the applied Ohmic electric field. In order to avoid the runaway problem, the applied Ohmic electric field has to be small. This leads us to the small- $E_{\|}$ordering $\hat{E}_{\|} \equiv\left(c E_{\|} / m w_{b}\right) \ll 1$, and we expand $f$ in $\hat{E}_{\|}: f=f_{a 0}+f_{\alpha 1}+f_{\alpha 2}+\cdots$. Then the H-theorem assures us a Maxwellian solution for the combined $f_{0}: f_{0}=j_{\text {to }}+f_{\mathbf{w}_{0}}=$ $f_{M}=$ Maxwellian , and

$$
\begin{aligned}
& f_{t 0}=f_{M} \Theta\left(\xi_{c}-|\xi|\right), \\
& f_{p 0}=f_{M} \Theta\left(|\xi|-\xi_{c}\right),
\end{aligned}
$$

where $\xi_{\mathrm{c}}$ is the magnitude of the pitch angle corresponding to the trappedpassing boundary, and $\Theta$ is the heavy-side step function. The first order version of Eq. (1) becomes, using Eq. (2):

$$
\vec{\eta}_{\|} \cdot \nabla f_{\alpha 1}-C\left(f_{a 1}\right)=-\frac{e E_{\|}}{T} \eta_{\mid l} f_{\alpha 0}+\frac{e E_{\| 1}}{m v}\left(1-\xi^{2}\right) \frac{\partial f_{a 0}}{\partial \xi} .
$$


The first term in the right-hand side of Eq. (5) represents the usual value of $\nabla \cdot \Gamma$ obtained by corisidering the trapped and passing particles as one Maxwellian species. Any divergence-free flow, no matter how large it is, cannot contribute to the first term and cannot be calculated from the drift kinetic equation if the trapped and passing particles are treated as one species. In determining the perturbed distribution function from the differential equation (drift-kinetic equation), the flux of particles across the trapped-passing boundary is often an important factor, due to the different properties between the trapped and passing particles. By considering the trapped and passing particles as two different species, such a particle exchange between the trapped and passing regions can be easily evaluated, as can be seen from the second term in the right-hand side of Eq. (5).

If the trapped and passing particles are treated as one species, such a driven flow will have to be calculated outside of the drift-kinetic equation and used as an additional boundary condition in determining $f_{1}$ at the trapped-passing boundary. This additional flow cannot be easily bandled within the framework of the usual variational approach ${ }^{1.2}$ if the trapped and passing particles are treated as one species, because the electrical force on the present Ohmically reversing particles are balanced by both collisions and bounce motions. This is unlike the usual cases, where there are clearcut relationships between the forces and the class of particles: the electric force on the passing particles is mainly balanced by collisons and on the trapped particles by bounce motions. Determination of the Ohmically reversing particle flow requires determination of the perturbed distribution 
function for the Ohmically reversing particles. However, in this situation, the attempt to describe the purturbed distribution function for the Ohmically reversing particles, within the one species model, can become highly tedious and erroneows. By treating the trapped particles and the passing particles as two separate species, we can easily determine the divergencefree, Ohmically reversing particle flow across the trapped-passing boundary, and incorporate it into the drift-kinetic equation as the second term in the right-hand side of Eq. (5). We can treat the second term as if it is an additional scattering term localized at the trapped-passing boundary. We note here that including the particle fiow across the trapped-passing boundary in this manner in the drift-kinetic equation is not usual, but mainly a matter of mathematical convenience.

Using Eqs. (3) and (4), we can write the first order equations as

$$
\begin{aligned}
& \vec{v}_{\| 1} \cdot \nabla f_{p 1}-C\left(f_{p 1}\right)=-\frac{e E_{\| l}}{T} \eta_{\|} f_{M}-\frac{e E_{\| 1}}{m} f_{M} \frac{2 \xi}{B v}\left(1-\xi^{2}\right) \delta\left(\lambda-\lambda_{c}\right), \\
& \vec{v}_{\|} \cdot \nabla f_{t 1}-C\left(f_{n 1}\right)=-\frac{e E_{\|}}{T} \eta_{\|} f_{M}+\frac{e E_{\|}}{m} f_{M} \frac{2 \xi}{B v}\left(1-\xi^{2}\right) \delta\left(\lambda-\lambda_{c}\right),
\end{aligned}
$$

where $\lambda_{e}=\lambda$ at $\xi=\xi_{c}$. It is more convenient to transform the above equations, using a scheme similar to one used in Ref. 1 ,

$$
\begin{aligned}
f_{a 1}= & -\frac{e}{T} f_{M} \int_{0}^{l} \frac{d \ell_{p}}{B_{p}}\left[B E_{\|}-\frac{B^{2}\left\langle E_{\|} B\right\rangle}{\left\langle B^{2}\right\rangle}\right] \\
& +v_{\|} f_{*} \frac{B\left\langle E_{\|} B\right\rangle}{\left\langle B^{2}\right\rangle}+H_{\alpha}, \quad \alpha=p \text { or } t,
\end{aligned}
$$

where the ion flow has been assumed to be zero for simplicizy, $\ell_{p}$ is the length of the field line in the poloidal direction, $\langle\ldots\rangle$ represents the flux-surface 
average, and $f_{\text {, is }}$ is the Spitzer function defined as $C\left(\eta_{\|} f_{s}\right)=(e / T) \eta_{\|} f_{M}$. Using the above transformation of $f_{\alpha 1}$ into $H_{a}$, we obtain

$$
\begin{aligned}
\vec{v}_{\|} \cdot \nabla H_{p}-C\left(H_{p}\right) & =-\vec{v}_{\|} \cdot \nabla v_{\|} f_{s} \frac{B<E_{\|} B>}{<B^{2}>}-\frac{e E_{\|}}{m} f_{M} \frac{2 \xi}{B v}\left(1-\xi^{2}\right) \delta\left(\lambda-\lambda_{c}\right), \\
\vec{v}_{\|} \cdot \nabla H_{t}-C\left(H_{t}\right) & =-\vec{v}_{\|} \cdot \nabla v_{\|} f_{t} \frac{B<E_{\|} B>}{<B^{2}>}-\frac{e E_{\|}}{m} f_{M} \frac{2 \xi}{B v}\left(1-\xi^{2}\right) \delta\left(\lambda-\lambda_{c}\right) .
\end{aligned}
$$

We can further simplify the above equations by defining $g$ and $g_{t}$ as follows:

$$
\begin{gathered}
C(g)=\frac{e E_{\|}}{m} f_{M} \frac{2 \xi}{B v}\left(1-\xi^{2}\right) \delta\left(\lambda-\lambda_{c}\right), \\
\vec{v}_{\|} \cdot \nabla g_{t}=-\frac{e E_{\|}}{m} f_{M} \frac{4 \xi}{B v}\left(1-\xi^{2}\right) \delta\left(\lambda-\lambda_{c}\right),
\end{gathered}
$$

and transforming $H_{\alpha}$ into $F_{\alpha}, H_{\alpha}=F_{\alpha}+g$. The first order equations are then finally transformed into

$$
\begin{aligned}
\vec{v} \cdot \nabla F_{p}-C\left(F_{p}\right) & =-\vec{v}_{\|} \cdot \nabla\left[v_{\| \mid} f_{\mathrm{a}} \frac{\left.B<E_{\|} B\right\rangle}{\left\langle B^{2}\right\rangle}+g\right], \\
\vec{v} \cdot \nabla F_{t}-C\left(F_{t}\right) & =-\vec{v}_{\|} \cdot \nabla\left[v_{\|} f_{t} \frac{\left.B<E_{\|} B\right\rangle}{\left\langle B^{2}\right\rangle}+g-g_{t}\right] .
\end{aligned}
$$

The function $g$ represents the effect of additional electron flux due to the Ohmically reversing motions, and thus $\left.\eta_{\|} f_{,} B<E_{\|} B\right\rangle /\left\langle B^{2}\right\rangle+g$ constitutes a modified Spitzer function.

In the berans regime of collisionality, we adopt the small collision frequency ordering, $\nu_{*} \equiv\left(\nu_{c} R q / v e^{3 / 2}\right) \ll 1$, where $q$ is the safety factor, $\epsilon \equiv r / R$ is the inverse aspect ratio, and $\nu_{c}$ is the electron $90^{\circ}$-scattering frequency. And we expand $F_{\alpha}$ in $\nu_{s}: F_{\alpha}=F_{\alpha}^{(0)}+F_{\alpha}^{(1)}+F_{\alpha}^{(3)}+\cdots$, where the superscripts represent the corresponding orders in $\nu_{\text {n. }}$. Then, to the 
lowest order in $\nu_{*}$, Eqs. (6) and (7) become

$$
\begin{aligned}
& \vec{v} \cdot \nabla F_{p}^{(0)}=-\vec{v}_{\|} \cdot \nabla\left(v_{\|} f_{0} \frac{\left\langle B E_{\|} B\right\rangle}{\left\langle B^{2}\right\rangle}+g\right), \\
& \vec{v} \cdot \nabla F_{t}^{(0)}=-\vec{v}_{\|} \cdot \nabla\left(v_{\|} f_{0} \frac{\left\langle B E_{\|} B\right\rangle}{\left\langle B^{2}\right\rangle}+g-g_{t}\right),
\end{aligned}
$$

and the solution $F_{\alpha}^{(0)}$ is trivially obtained as follows:

$$
\begin{aligned}
& F_{p}^{(0)}=-v_{\|} f_{1} \frac{\left.B<E_{\|} B\right\rangle}{\left\langle B^{2}\right\rangle}-g+G_{p}, \\
& F_{t}^{(0)}=-v_{\|} f_{x} \frac{\left.B<E_{\|} B\right\rangle}{\left\langle B^{2}\right\rangle}-g+g_{t}+G_{t},
\end{aligned}
$$

where the function $G_{\alpha}$ is independent of the poloidal angle $\theta$ and is determined from the first order(in $\left.\nu_{*}\right)$ version of Eqs. (6) and (7) in the form of a solubility condition

$$
\left\{C\left(F_{\alpha}^{(0)}\right)\right\}_{b}=0,
$$

where $\{\cdots\}_{b}$ represents the bounce average operator.

The function $H_{a}^{(0)}$ is then given by the relation $H_{\alpha}^{(0)}=F_{\alpha}^{(0)}+g$, and thus

$$
\begin{aligned}
& H_{i}^{(0)}=-v_{\|} f_{t} \frac{\left.B<E_{\|} B\right\rangle}{\left.<B^{2}\right\rangle}+G_{p}, \\
& H_{t}^{(0)}=-v_{\| f_{t}} \frac{\left.B<E_{\|} B\right\rangle}{\left\langle B^{2}\right\rangle}+g_{t}+G_{t} .
\end{aligned}
$$

And the current density is calculated as:

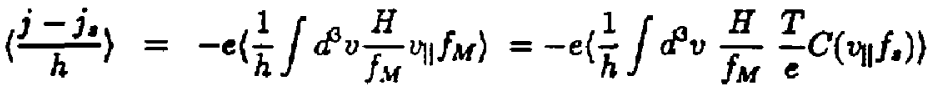

$$
\begin{aligned}
& \cong-T\left(\frac{1}{h} \int d^{3} v \text { v } \frac{f_{3}}{f_{M}} C\left(H^{(0)}\right)\right)
\end{aligned}
$$


where the self-adjointness property of the Coulomb collision operator has been used and $j$, is the Spitzer current density defined as

$$
\left\langle\frac{j_{*}}{h}\right\rangle=-e\left(\frac{1}{h^{2}} \int d^{3} v v_{\|}^{2} f_{*}\right\rangle \frac{\left.B_{o}<E_{\|} B\right\rangle}{\left\langle B^{2}\right\rangle} .
$$

The expression for $H_{p}^{(0)}$ and $H_{t}^{(0)}$ are :ien to be substituted into the above equation. However, from the definition of $g_{t}$ and $G_{t}$, it can be easily seen that $g_{t}$ and $G_{l}$ are even functions of $v_{\|}$and, therefore, cannot contribute to $\left\langle\left(j-j_{\text {s }}\right) / h\right\rangle$. Thus our neoclassical current density becomes

$$
\left(\frac{j-j_{e}}{h}\right) \cong-T\left(\frac{1}{h} \int d^{3} v v_{\|} \frac{f_{*}}{f_{M}} C\left[-v_{\|} f_{0} \frac{\left.B<E_{\|} B\right\rangle}{\left\langle B^{2}\right\rangle}+G, \Theta\left(\lambda_{c}-\lambda\right)\right]\right) \text {. }
$$

It remains to determine the function $G_{p}$ from the constraint equation (8):

$$
\left\{C\left(-v_{\|} f_{*} \frac{B<E_{\| l} B>}{\left.<B^{2}\right\rangle}+G_{p}\right)+\frac{e E_{\| 1}}{m} f_{M} \frac{2 \xi}{B v}\left(1-\xi^{2}\right) \delta\left(\lambda-\lambda_{c}\right)\right\}_{b}=0 .
$$

By subtracting out $G_{0}$, the known part of $G_{p}$ from Ref. 1, which satisfies

$$
\left\{C\left(-v_{\|} f_{0} \frac{\left.B<E_{\|} B\right\rangle}{\left\langle B^{2}\right\rangle}+G_{0}\right)\right\}_{b}=0
$$

we obtain

$$
\left\{C\left(G_{p}\right)+\frac{e E_{11}}{m} f_{M} \frac{2 \xi}{B v}\left(1-\xi^{2}\right) \delta\left(\lambda-\lambda_{c}\right)\right\}_{*}=0,
$$

where $G_{p}=G_{2}-G_{0}$. Since a discontinuous structure of the second derivative of $G_{;}^{*}$ in $\lambda$ is responsible for the $\delta$-function, we approximate the collision operator as a simplified pitch angle scattering operator in this letter:

$$
C\left(G_{p}^{*}\right) \equiv \nu_{e} \frac{2 \xi}{B} \frac{\partial}{\partial \lambda} \xi \lambda \frac{\partial}{\partial \lambda} G_{p}^{*} \simeq \nu_{e} \frac{2 \xi^{2} \lambda}{B} \frac{\partial^{2}}{\partial \lambda^{2}} G_{p}^{*}
$$


where $\nu_{e}=\nu_{e i}+\nu_{e e}$ contaias both electron-ion collision frequency $\nu_{e i}$ and the electron-electron collision frequency $\nu_{\text {ee. }}$ Then Eq. (10) trivially yields

$$
C\left(G_{p}^{*}\right) \simeq-\frac{\left.2 e<E_{\|} B\right\rangle}{m v B} \lambda \frac{\xi^{2}}{\langle\xi>} f_{M} \delta\left(\lambda-\lambda_{c}\right) .
$$

Equation (9) then becomes

$$
\begin{aligned}
& \left\langle\frac{j-j_{s}}{h}\right\rangle \cong-T\left\langle\frac{1}{h} \int_{\mathrm{pm}} d^{3} v v_{\|} \frac{f_{s}}{f_{M}} C\left[-\frac{v_{\|} f_{0}}{h} \frac{\left.B_{0}<E_{\|} B\right\rangle}{\left\langle B^{2}\right\rangle}+G_{o}+G_{p}^{-}\right]\right\rangle \\
& =\sigma_{0} \frac{B_{0}\left\langle E_{\| \mid} B\right\rangle}{\left\langle B^{2}\right\rangle}-T\left\langle\frac{1}{h} \int d^{2} v v_{\|} \frac{f_{0}}{f_{M}} C\left(G_{p}^{*}\right)\right\rangle \\
& \cong \sigma_{0} \frac{\left.B_{0}<E_{\|} B\right\rangle}{\left\langle B^{2}\right\rangle} \\
& +\frac{2 T_{e}<E_{\|} B>}{m<\xi_{c}>}\left\langle\frac{1}{h B} \int \frac{d^{d} v}{v} \lambda \xi^{2} v_{||} f \delta \delta\left(\lambda-\lambda_{c}\right)\right\rangle .
\end{aligned}
$$

Here $\sigma_{\circ}$ is the usual neoclassical reduction amount from the Spitzer con-

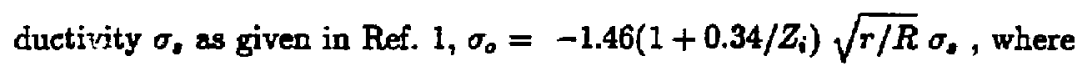
$Z_{i}$ is the ionic charge number. By evaluating the velocity integral and the flux-surface-average in a straightforward manner, and using the numerical tabulation of Ref. 4 for $f_{s}$, we find that the second term in $\left\langle\left(j-j_{s}\right) / h\right\rangle$ becomes

$$
0.387\left(1+\frac{0.413}{Z_{i}}\right) \sqrt{\frac{T}{R}} \sigma_{0} \frac{B_{0}\left\langle E_{i \mid} B\right\rangle}{\left\langle B^{2}\right\rangle},
$$

where in the parentheses, the first term is from $\nu_{e i}$ and the $Z_{\mathrm{i}}$-dependent term is from $\nu_{\infty}$ (the $1 / Z_{i}$ dependence is an approximate fit).

Combining the above two terms, we finally obtain

$$
\left\langle\frac{j}{h}\right\rangle=\left[1-1.07\left(1+\frac{0.32}{Z_{i}}\right) \sqrt{\frac{r}{R}}+\left(0.07+\frac{0.34}{Z_{i}}\right) \frac{r}{R}\right] \sigma, \frac{\left.B_{o}<E_{\|} B\right\rangle}{\left\langle B^{2}\right\rangle},
$$


where the term proportional to $r / R$ is added here to make $\langle j / h\rangle \rightarrow 0$ as $r / R \rightarrow 1$. As a conclusion, the electrical conductivity in the banana regime calculated here, modified by the electrostatic trapping effect in the applied Ohmic field, is significantly enhanced from the previous result. For $Z_{i}=1$, the conductivity values are about $28 \%$ closer to the value of Spitzer and Hürm. 4

\section{Acknowledgements}

The author thanks Dr. W. Lawson of NYU for valuable discussions. This work was supported by U.S. Department of Energy under the contract numbers DE-FG02-86-ER53223 and DE-AC02-76CH03073. 


\section{References}

${ }^{1}$ F.L. Hinton and R.D. Hazeltine, Rev. Mod. Phys. 48, 239 (1976).

${ }^{2}$ M.N. Rosenbluth, R.D. Hazeltine, and F.L. Hinton, Phys. Fluids 15, 116 (1972).

${ }^{3}$ S.P. Hirshman, R.J. Hawryluk, and B. Birge, Nucl. Fusion 17, 611 (1977).

'L. Spitzer, Jr. and R. Härm, Phys. Rev. 89, 977 (1953). 


\section{Figures}

FIG. 1. Trapped-passing boundaries in $\left(v_{\|} / v\right)$ and poloidal angle space. (a) Boundarieg without the modification by the applied electric field. (b) Boundaries with the modification. Eilectrons in the shaded region are Ohmically reversing. And the electrons in the dotted area are truely trapped.

FIG. 2. Exaggerated illustion of the Ohmically reversing electron orbits in $(\lambda, \sigma, \theta)$-space. The dotted areas are the usual magnetically trapped regions. 

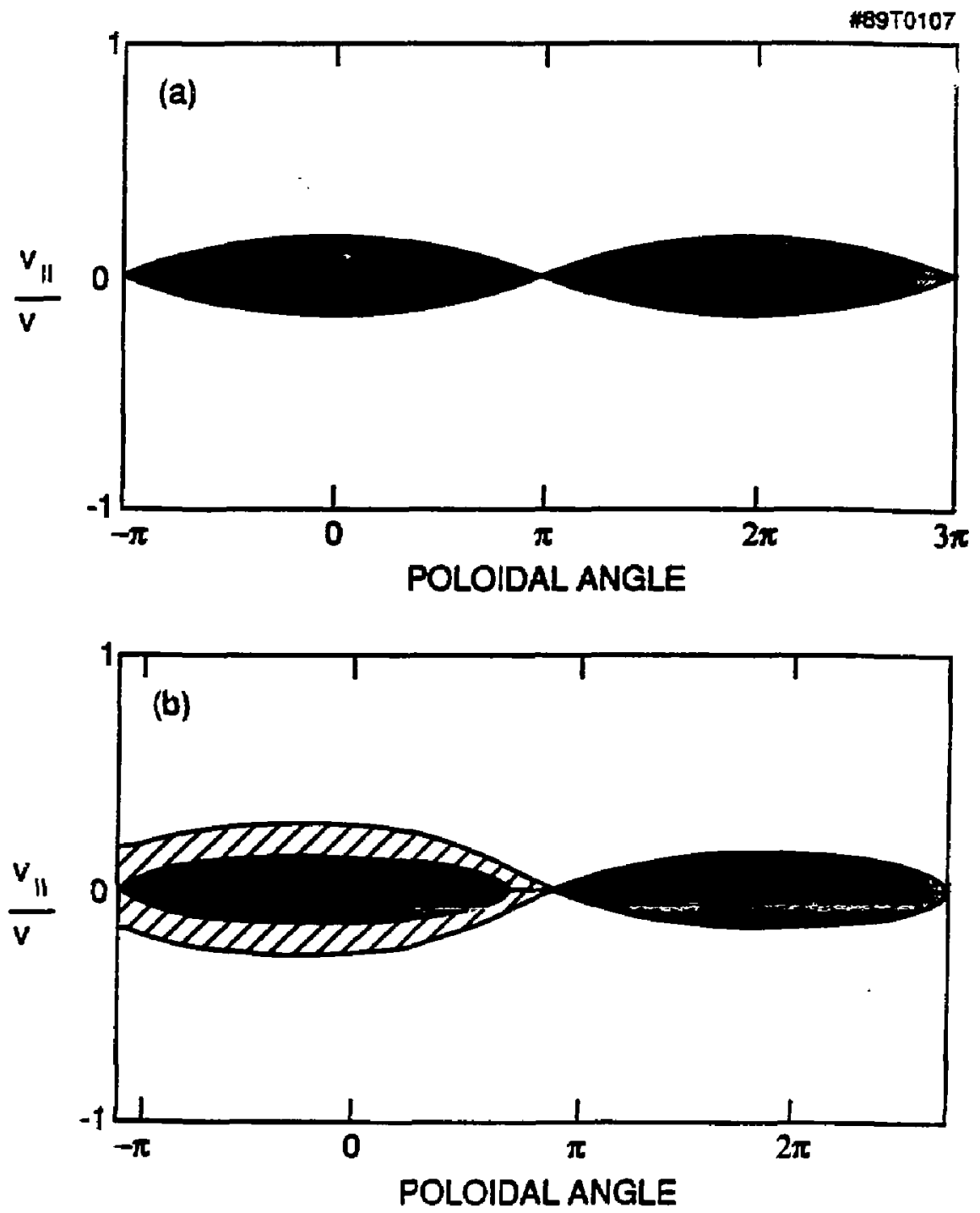

Fig. 1 
\$89T0108

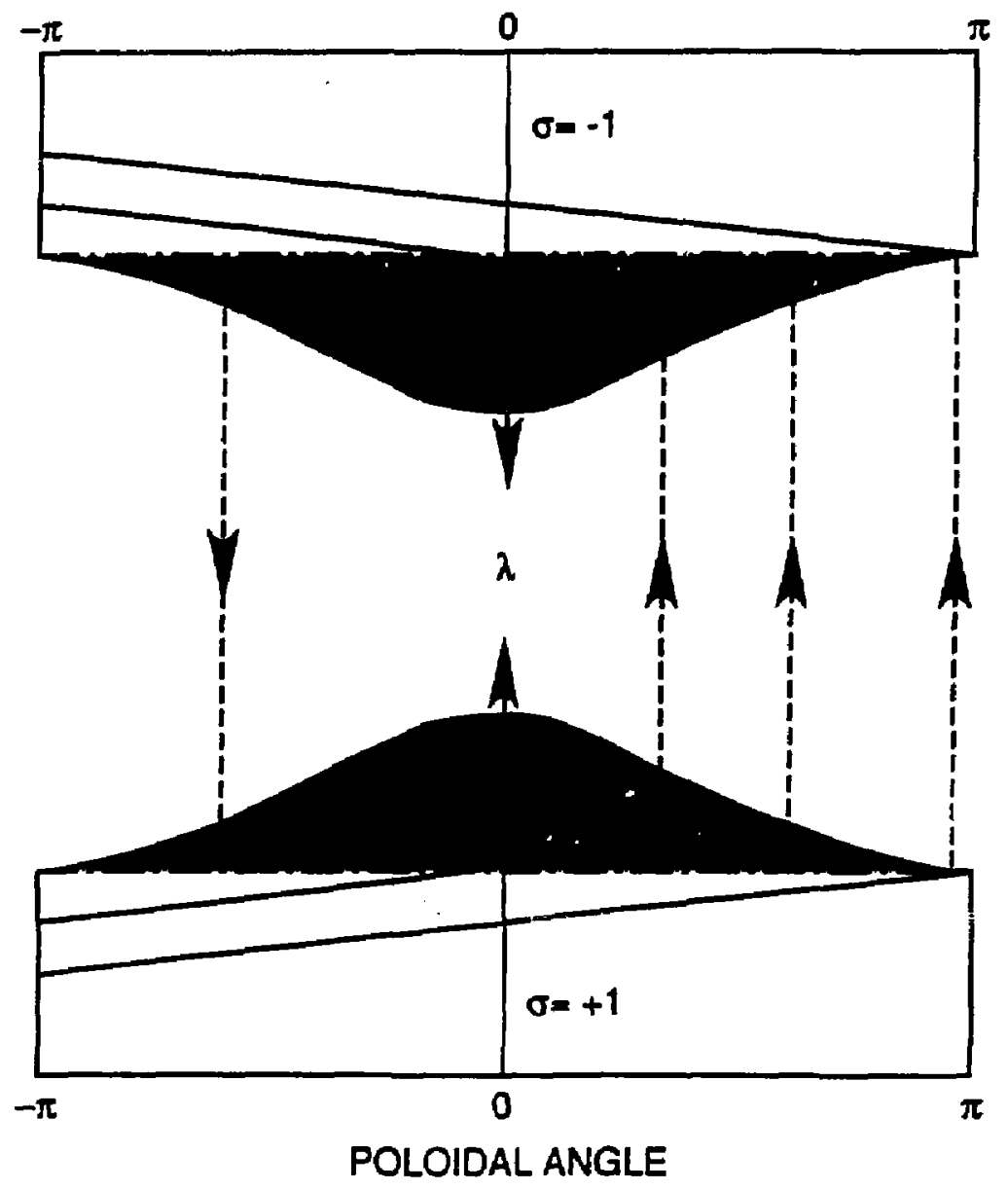

Fig. 2 
Dr. Frank J. Paolonl, Univ of wollongong, AUSTRALIA

Prof. M.H. Bronn.-n, Univ Sydney, AuSTRALIA

Piasag Research Lab.. Australian Mat. Univ.., AUSTRALIA

Prot. I.R. Jones, Flinders Univ., AUSTRaLIA

Prof. F. Cap, Inst Theo Pnys, AustRIA

Prof. M. Hindler, instut fur Theoretische Physik, AuSTRIA

M. Goossens, Astronadiseh instltuut, BELGIL

Ecole Royle Hilitelre, Lo a Phys Plasasa, Belgiun

Comiselon-European, Ds-XII Fusion Prog, BELGILM

Prot, R. Bouclque, RIJksun I versitalt Gant, BELGIUA

Dr. P.H. Sakarraka, Instltuto Fisica, EarziL

Instituto Do Pesquisas Espaciasi-IRPE, BRill

Docueants oftice, Atomic Energr of Conede Limited, Cawah

Or. H.P. Bachynski, was Teehnologles, Inc., Curad

Or. H.M. Skeragard, University of Sesketehowan, Cuwan

Dr. H. Earnard, Univertity of British Colubia, Cawad

Prof. J. Tolchann, Univ. of Montreal, caupa

Prof. S.R. Sreanivanen, Univers!ty of Calgary, cawoh

Prof. Tudor W. Johnstoa, Iers-Enargit, cuwan

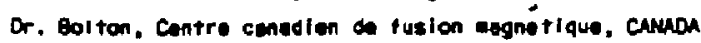

Dr. C.R. Jases, Unive of Albarta, canch

Dr. Poter Lukec, Komenskeho UnIv, CzEChoslovakia

The Librerien, Culne Leorotory, EkELAN

The Librarlan, Autherford Appiaton Laboratory, ENGLA

wrs. S.A. Hutchinson, JET Library, EKGLAND

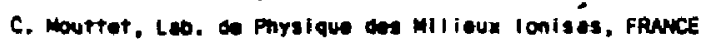

d. Radat, CENCADurache - ant sas, FRuce

Ms. C. RInnl, Librerien, Univ, of loannina, GeEECE

Dr. Tom Mal, Acadeay Blbilicgraphic Sar., horw kong

Preprint Librery, Hengerian Mcediry of Sciences, Huncapy

Or. B. Das Gupte, Sawe Inst of Mucl. Phys., INola

D. P. Kew, Instltute for Places Raseareh, INDIA

Dr. Philid Posensu, isreel inst. of Tech, ISRAEL

Librerien, Int'' Cte Theo Phrs, ITALY

Prof. G. RostegnI, Istituto Gas Ionizzati Del CAR, ITALY

Miss cielis os Polo, Assoc EuRATOH-ENEA, ITALY

Or. G, Growso. Igtituto di Flsica del Plasag, ITALY

Or. H. Yeato, Toshl be Res 1 Oer, JNPAY
Prot. 1. Kowakomi, Atomic Enargy Res. Instituto, JAPAN

Prot. Kyoji Nishikawa, Univ of Hiroshima, JAPAN

Director, Dept. Large Tokamak Res JAERI, JAPAN

Prot. Satosti Itoh, Kyushu University, JAPAN

Resuarch info Conter, Nogoya University, JAPAN

Prof. \$. Tanoke, Kyoto University, JAPAN

Library, Kyoto University, JAPAN

Prof. Nobuyukl Inowe, Univergity of Tokyo, JAPAN

5. WOS $i$, JAERI, JAPAN

H. Jeong, Llbrarian, Kores Advanced Energy Res inst, KCREA

Prof. D.I. Chol, The Korea Adv, Inet of Sel a Tech, KoneA

Prof. B.S. Llley, University of maikato, MEW ZEALAND institute of Plase Physies, PECPLE's Repual ic of CHIM Librarian, Institute of Phys., PECPLE's RePualic of CHINA Library, Tsing the University, PEOPLE'S REPLalic of CHINA 2. Li, Southwast inst. Physics, PeOrel's Replatic of CHik Prof. J.A.C. Cabrel, Inst Suderior Teenico, Pontuall

or. Octavian Petrus, AL I CuZA Univarsity, Rowawia

Dr. Jae villiers, Fusion Studies, AEC, SO AFRICA

Prot. M.A. Il iberg, University of Maral, SO AFRICa

C.I.E.H.A.T., Fusion Div. Library, SPAIN

or. Lennart Stenflo, University of UEEA, SWEDEH

Librory, Royel Institute of Teen, SwEDEN

Prot. Hens Withetwion, Chaluars Univ of Tech, SwEDEN

Centre Phys des PIasase, Ecole Polytech Fed, SWITZERLAND

siollotheak, fom-inst voor PI Isad fysico, ThE RETHERLANOS

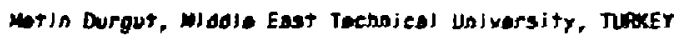

Dr. D.D. Ryutov, Sibarian Acad Scl, USSR

Dr. G.A. El iseev, Kurchutov Institute, USSR

Dr. Y.A. Glukhikh, Inst Electrophysleal Apparatus, USSR

Prot . Q.5. Podichenko, Inst. of Phys. B Tech, USSR

Dr. L.M. Kovrizhnykh, Institute of Gan. Physies, USSR

Nuclear Ros. Establishent, Julich Ltd., W. GERMAY

Bloliothak, Inst. Fur Plasnaforser.- -9, W. GERwwY

Or. K. Sehindler, Ruhr-Universltat Bochum, W. GEPanky

ASOEX Roading Ro, clo Wegner, IPPMax-Planck, W. GERauNY

Librarian, Max-ianck institut, W. GrRwayr

Prot. R.K. Jomov, inst of Phys, nugoslavia 